Initiale Kombinations-Therapie auch bei mäßigen BPH-Beschwerden

- Das Prostatavolumen ist ein nach wie vor unterschätzter Parameter im Management von Patienten mit einem benignen Prostatasyndrom (BPS). Bereits bei moderaten Symptomen des unteren Harntraktes (LUTS), einem Prostatavolumen von $\geq 30 \mathrm{ml}$ und einem PSA-Wert von $\geq 1,5 \mathrm{ng} / \mathrm{ml}$ sollte die Behandlung aufgrund der aktuellen Evidenzlage und laut Leitlinien mit einer Kombination aus dem Alpha-Blocker Tamsulosin plus dem 5a-Reduktasehemmer (5-ARI) Dutasterid $\left(\right.$ Duodart $\left.^{\oplus}\right)$ begonnen werden, berichtete PD Dr. Christian Gratzke, München. Der Nutzen einer initialen Kombinationstherapie mit Tamsulosin/Dutasterid wurde in der CombAT (Combination of Avodart ${ }^{\oplus}$ and Tamsulosin)Studie bei 4.844 Patienten $\geq 50$ Jahren mit moderaten bis schweren BPH-Symptomen (International Prostate Symptom Score, IPSS $\geq 12$ ), einem Prostatavolumen $\geq 30 \mathrm{ml}$ und einem PSA $\geq 1,5 \mathrm{ng} / \mathrm{ml}$ gezeigt [Roehrborn CG et al Eur Urol 2010; 57: 123-31].

Nach vier Jahren schnitt die Kombinationsgruppe sowohl in Bezug auf die Symptomverbesserung als auch auf die Reduktion des Komplikationsrisikos signifikant besser ab als die Alpha-Blocker-Monotherapie. Das relative Risiko für einen akuten Harnverhalt oder BPHbedingte Operationen sank unter Tamsulosin/ Dutasterid um 65,8\% im Vergleich zu einer Tamsulosin-Monotherapie ( $p<0,001$ ).

Neue Daten einer retrospektiven Studie bestätigen die CombAT-Daten und zeigen einen über zehn Jahre anhaltenden Benefit der
Kombinationstherapie mit Alpha-Blocker/ 5-ARIs. Darunter sank das Risiko für einen akuten Harnverhalt um $79,4 \%$ und für einen chirurgischen Eingriff um $61,9 \%$ gegenüber einer Alpha-Blocker-Monotherapie.

Im Gegensatz zu einer initalen Kombinationstherapie erhöht die initiale Gabe eines AlphaBlockers und die verzögerte Gabe eines 5-ARI laut Gratzke das Komplikationsrisiko und führt dazu, dass die Progression des BPS verzögert gehemmt würde.

Abdol A. Ameri

Satellitensymposium „Moderne Versorgung von Patienten mit benignem Prostatasyndrom (BPS) - eine kritische Analyse" im Rahmen des 64. Kongresses der Deutschen Gesellschaft für Urologie,

Leipzig, 27. September 2012;

Veranstalter: GlaxoSmithKline, München

\section{MCRPC: Abirateronacetat auch bei chemotherapienaiven Patienten effektiv}

\begin{abstract}
- Abirateron, der aktive Metabolit von Abirateronacetat (Zytiga $\left.{ }^{\circledR}\right)$, unterdrückt die Androgen-Biosynthese in allen endokrinen Organen einschließlich Hoden, Nebenniere und auch im Tumorgewebe selbst. In der zulassungsrelevanten Phase-III-Studie COU-AA-301 bei Patienten mit metastasiertem kastrationsresistentem Prostatakarzinom ( $\mathrm{mCRPC}$ ), deren Erkrankung während oder nach Standardchemotherapie mit Docetaxel progredient geworden ist, führte Abirateronacetat im Vergleich zu Placebo (jeweils in Kombination mit Prednison/Prednisolon) zu einer signifikanten Verbesserung des Gesamtüberlebens [de Bono JS et al. N Engl J Med 2011; 364: 1995-2005]. Die kürzlich vorgestellten Interimsdaten der COU-AA-302-Studie [Ryan CJ et al. J Clin Oncol 30, 2012 suppl; abstr LBA4518] deuten darauf hin, dass die Substanz auch in der chemotherapienaiven Situation Placebo überlegen ist, berichtete Prof. Kurt Miller, Berlin. In die randomisierte, doppelblinde Phase-III-Studie wurden 1.088 asymptomatische oder nur mild symptomatische, chemonaive MCRPC-Patienten eingeschlossen und entweder mit Abirateronacetat $(1.000 \mathrm{mg} / \mathrm{Tag})$ plus Prednison $(5 \mathrm{mg}$ zweimal täglich) oder Placebo plus Prednison behandelt. Die mediane Nachbeobachtungszeit betrug 22,3 Monate. Die Verumgruppe war sowohl in Bezug auf den primären Endpunkt, dem radiografisch bestimmten progressionsfreien Überleben (rPFS), der Placebogruppe signifikant überlegen als auch in Bezug auf die sekundären Endpunkte wie die Zeit bis zum Opioideinsatz, bis zum Beginn der Chemotherapie, bis zur ECOGVerschlechterung sowie die Zeit bis zur PSA-Progression. Beim Gesamtüberleben ergab die Interimsanalyse einen starken Trend für eine Überlegenheit von Abirateronacetat plus Prednison. Während das mediane Gesamtüberleben im Verumarm noch nicht erreicht wurde, lag es im Placebo-Arm bei 27,2 Monaten. Abdol A. Ameri
\end{abstract}

Fachpresskonferenz „Zytiga ${ }^{\oplus}$ : Therapie des metastasierten kastrationsresistenten Prostatakarzinoms im Wandel“ im Rahmen des 64. Kongresses der Deutschen Gesellschaft für Urologie, Leipzig, 27. September 2012,

Veranstalter: Janssen-Cilag, Neuss

\section{Goldene Tablette ${ }^{\oplus}$ für Astellas Pharma}

_ Zum fünften Mal hat Astellas Pharma den Oscar der Gesundheitsbranche erhalten. Das Unternehmen nahm im September in München die Goldene Tablette ${ }^{\circledast}$ im Bereich Urologie entgegen. Rund 1.500 Ärzte aus zehn verschiedenen Fachrichtungen stimmten im Auftrag der Zeitschrift PharmaBarometer zum 13. Mal über ihre Favoriten in den Kategorien Außendienst, Service, Produktqualität, Preis-Leistungsverhältnis und Rabattverträge sowie über das Image der Arzneimittelhersteller ab.

Die Goldene Tablette ${ }^{\oplus}$ gehört zu den bedeutendsten Auszeichnungen im Gesundheitswesen. „Die Wahl zeigt das große Vertrauen der Ärzte in unsere Produkte und ihre Zufriedenheit mit unserem Außendienst. Sie spornt uns gleichzeitig an, den Verpflichtungen und Anforderungen der Auszeichnung nachzukommen und unsere Position als führendes Pharmaunternehmen in der Urologie zu festigen." sagte Eberhard Karle, Director Sales bei Astellas Pharma. Er erklärte, dass in diesem Bereich derzeit die meisten neuen Produkte entwickelt werden.

Nach Informationen von

Astellas, München 